\title{
Biotechnology and Managed Care
}

\author{
by August J. Salvado and Grant Lawless
}

$\mathrm{T}$ he twentieth century witnessed an explosion in human inventiveness, including aviation, the transmission of sound and data by wireless communication, and the development of the semiconductor and digital computing. Recombinant deoxyribonucleic acid (DNA), which unraveled the mysteries of the biological sciences, was also an important discovery. Although each of these technologies continues to have profound effects on human existence, none will do so in a greater variety of ways than the field of biotechnology. Virtually everything that is important to health care practitioners and patients (e.g., diagnostic techniques, disease pathology, prevention, treatment, medical education, health care facility design, and legal and ethical issues) will be fundamentally transformed by the current revolution in biotechnology and genetic medicine.

Biotechnology is the application of biology to the development of products and services that use naturally occurring molecules created to restore biologic processes.' Biotechnology has evolved dramatically during the past 50 years, beginning with the discovery of DNA and its structure in the 1950s, the identification of its genetic code in the mid-1960s, and the cloning of the first human gene, somatostatin, in 1977 (Figure 1). ${ }^{2}$ In the early 1980s, the ability to manufacture
DNA using recombinant technology opened up many commercial possibilities. In 1.982, for example, recombinant human insulin was approved for use in refractory diabetics. ${ }^{3}$ Since then, biotechnology has progressed exponentially.

This article presents an overview of the biotechnology industry, with a discussion of new techniques and their relationships to drugs that are either in the lesting phase or approved for human use. Patient care and financial implications of the biotechnology explosion for the managed care pharmacist also are discussed, including the recommended phases of a strategic planning process for formulary and medical-policy decision making. Finally, we look at future trends in biotechnology.

\section{The Current Biotechnology Industry}

Enthusiasm for the development of biotechnologic techniques and products led to substantial funding of the biotechnology industry in the late 1980s and early 1.990s. However, the late 1990s saw a decline in this funding, based on the perception that ventures were too risky, slow, or difficult. Despite variable patterns of success, the biotechnology industry has grown into a significant economic force, worth more than $\$ 13$ billion in annual revenue and encompassing more than 1,300 companies. ${ }^{1}$ Collectively, these biotechnology companies have
2,200 products in development; 1,700 of these products are in clinical trials, and more than 300 products are in late clinical trials (Table 1). ${ }^{+}$About one-third of the products in these trials are oncologyrelated. Other therapeutic categories most likely to benefit from biotechnology include hematology, infectious disease, immunology, human immunodeficiency virus (HIV)/acquired immunodeficiency syndrome (AIDS), rheumatology, and gastroenterology.

The Food and Drug Administration (FDA) has facilitated more rapid availability of biotechnology products in the marketplace-over the past decade, new drug application (NDA) review periods for biotechnology products decreased from more than 30 months to less than 12 months. This trend in accelerated regulatory review and approval time of biotechnology products is expected to continue.

\section{New Drug Discovery}

Some conventional therapies, such as cancer chemotherapy, are inadequate because of the lack of selectivity between dysfunctional and normal cells. The discovery of novel agents that will selectively target dysfunctional cells without causing toxicity to normal tissue has been fueled by the unveiling of a host of potential molecular targets through the application of molecular biology methods. These methods also

\section{Authors}

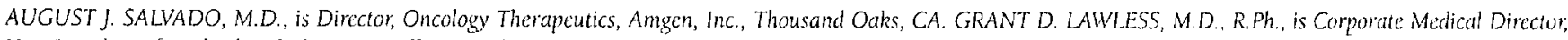
Vice President of Medical and Pharmacy Affairs, Highmark Blue Cross Blue Shield, Pittsburgh, PA.

AUTHOR CORRESPONDENCE: Augusl J. Salvado, M.D., Director, Oncology Therapeutics, Amgen, linc., One Amgen Center Drive, MS 35-1-B, Thousand Oaks, CA 91320; Tel: 805-447-2370; Fax: 805-499-9346; E-mail: asalvado@amgen.com.

ACKNOWLEDGEMENT: Sponsored by an uncstricted educational grant from Amgen, Inc.

Copyright@2000, Academy of Managed Care Pharmacy, Inc. All rights reserved. 


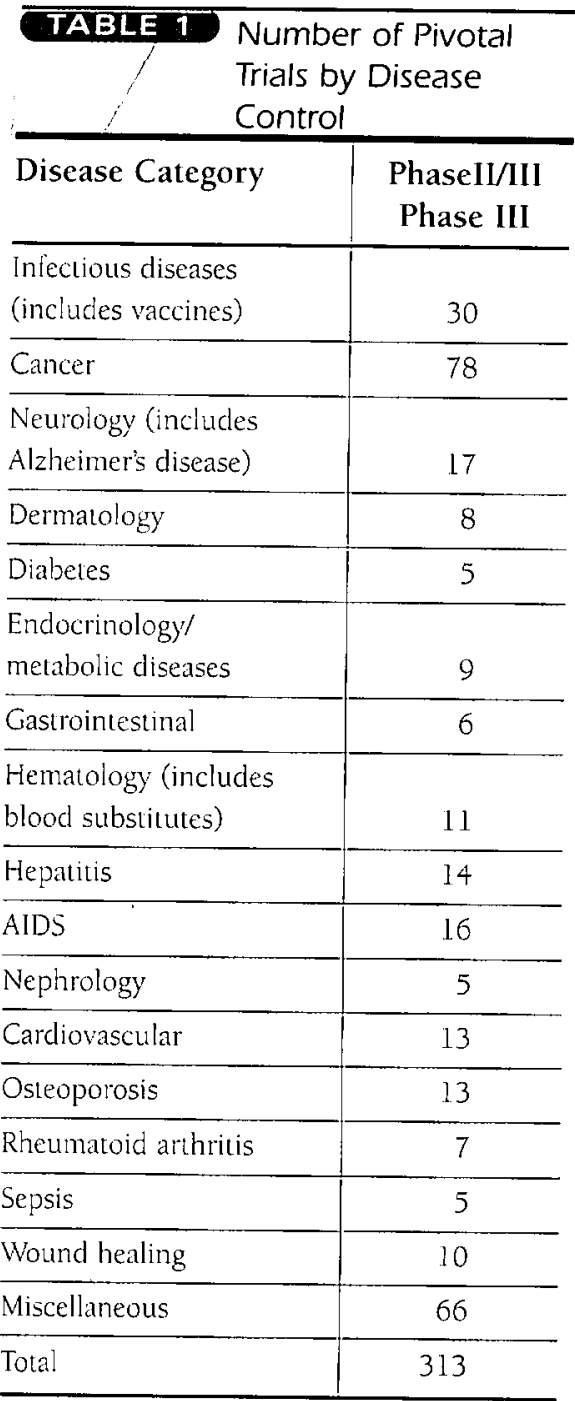

Source: Goldman-Sachs \& Co., Bio World Financial Watch, Ernst \& Young, LLP

have encouraged investigating these potential targets for drug discovery, by allowing functional expression or production of the targets for use in high-throughput screening assays of natural and synthetic molecule libraries. Interesting and creative approaches to treating diseased or dysfunctional cells have emerged.

\section{Genomics}

Gene therapy, the most important emerging area of biotechnology directly related to human health, may revolutionize modern medicine.' This important facet of biotechnology is based on genomics-

\section{FIGUAE 1 Evolution of Biotechnology}

\section{Evolution of Biotechnology}

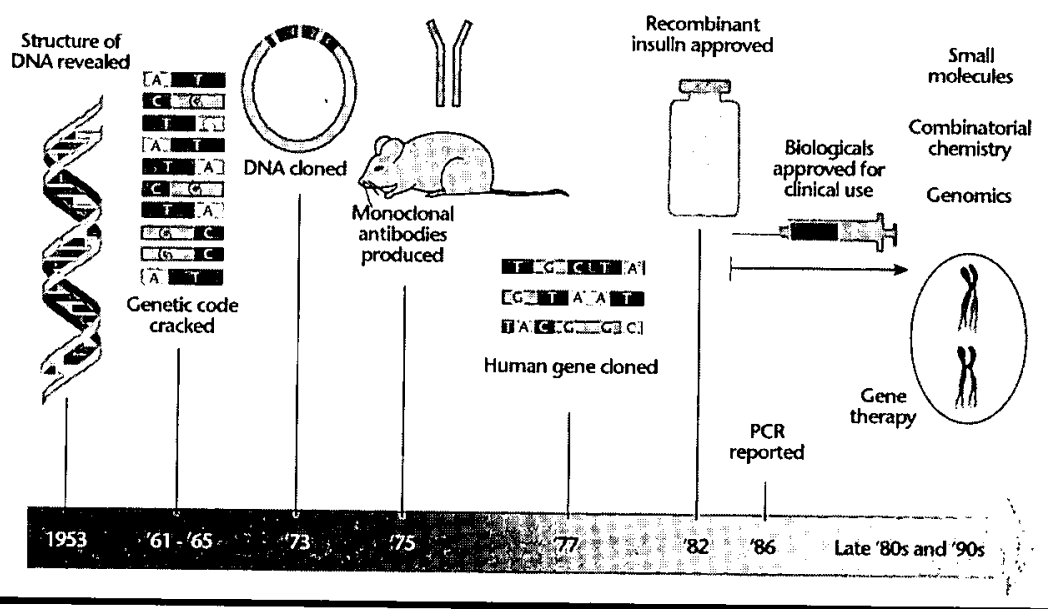

the study of genes and how they affect the human body. Although the term genomics is used broadly to categorize several types of biologic methods for research and drug development, in its most restricted definition it refers to the sequencing of DNA. ${ }^{5}$ The entire human genome (i.e., the full set of genes in an individual) is thought to consist of approximately 50,000-150,000 functional genes. A complete genome sequence contains the code for every structural protein and enzyme required for the synthesis of cellular components. ${ }^{6}$ The Human Genome Project, a government-funded consortium, is currently in a highly visible race with private industry to complete the entire sequence of the human genome within the next year or two. This remarkable effort will sort the entire code of three billion nucleotide base pairs and allow further efforts to identify disease-relevant genes.

To date, two biotechnology firms have sequenced approximately one-third of the three billion nucleotide-based pairs of the human genome. Computerized database searches for sequence similarities among gene sequences have yielded more important discoveries, and the role of genetics in many diseases has become more apparent.
Gene therapy applies the principles of genomics to the search for improvements in disease diagnosis, treatment, and prevention. Generally, gene therapy is characterized by the transfer of genetic information in the form of nucleic acid (usually DNA, which encodes for a therapeutic protein) into the cells of a patient for amelioration of disease. ${ }^{8}$ The goal of gene therapy is actually twofold: (1) to repair underlying genetic defects responsible for a number of disorders (e.g., hemophilia, cystic fibrosis); and (2) to overcome limitations, including low bioavailabilities, inadequate pharmacokinetic profiles, and high manufacturing costs often associated with the administration of traditional therapeutic proteins. ${ }^{9}$ Of the therapeutic opportunities for successful genetic intervention, oncology holds the most promise, with cancer gene therapy accounting for approximately $65 \%$ of gene-therapy clinical trials. ${ }^{9}$ Other potential uses for gene therapy are in hematology, immunology, pulmonology, and cardiology.

With few exceptions, the impact of genomics and other new technologies on pharmaceutical discovery has not been fully realized. However, the drug discovery process involving genomics is expected to evolve as more molecular drug targets are extracted from gene sequencing data. 


\section{- Therapeutic Applications of Biotechnology}

\section{Monoclonal Antibodies}

Monoclonal antibodies (MAbs) are specialized forms of protein, designed to target other proteins, enzymes, or receptors that are elevated during disease. MAbs are used to facilitate delivery of drugs or toxins to pathologically altered cells and to carry enzymes to tumor surfaces to activate pro-drugs. Additionally, radiolabeled MAbs are used for site-directed delivery of radioisotopes.

\section{Signal Transduction Inhibitors}

Recently, a better understanding of the molecular basis of cellular communication indicates that a number of diseases result from a malfunction of intracellular signaling. The activity of a particular signal transduction pathway is often enhanced or inappropriately active in the diseased cell compared with healthy cells; study results suggest that blocking a signaling element that is overactive in a tumor cell, but essential for normal cell function, is a promising therapeutic approach. ${ }^{10}$

\section{Matrix Metalloproteinase Inhibitors}

Matrix metalloproteinases (MMPs) are a gene family of at least 15 structurally related enzymes responsible for the degradation of extracellular matrix components associated with angiogenic and metastatic processes. The proteolytic activity of MMPs is normally regulated by the tissue inhibitors of metalloproteinases (TIMPs); disturbance of the MMP-TIMP balance can result in pathologies, such as rheumatoid arthritis, osteoarthritis, and atherosclerosis, as well as tumor growth and metastasis." MMP overexpression has been shown in prostate, lung, breast, and colon cancers and is five times higher in low-graded kidney tumor tissue compared with normal tissue. ${ }^{12}$

\section{Antisense Oligonucleotides}

Antisense oligonucleotides are a novel class of therapeutic agents used in the prevention and treatment of gene-mediated disorders. This class of compounds was developed on the premise that inhibiting the process and translation of messenger RNA blocks the expression of target genes involved in pathologic processes.

Antisense oligonucleotide therapy is being applied to oncology and hematology, and cardiovascular, infectious, and viral diseases. ${ }^{13}$ The first antisense-based drug marketed in the United States, fomivirsen sodium, has been approved for local treatment of cytomegalovirus retinitis in patients with AIDS. Phosphorothioate oligonucleotides being evaluated in phase I and II trials for the treatment of cancer and viral infections have demonstrated acceptable pharmacokinetic and safety profiles. ${ }^{13}$

\section{Angiogenesis Inhibitors}

Realization that tumors depend on a blood supply to both grow and metastasize fueled research for a method to eliminate the vasculature required to nourish growing tumors. The strategy behind angiogenesis inhibition represents a promising and unique breakthrough in cancer-research. Tumor angiogenesis involves a number of complex processes, beginning with the production and release of angiogenic factors by tumor cells or their surrounding matrix, which activates endothelial cells and culminates in a highly vascularized tumor. ${ }^{14}$

\section{- Preparing for the Biotechnology Explosion-Impact on the Managed Care Pharmacist}

The exceptional rate at which new pharmaceutical and biotechnology. products and applications are emerging is staggering. The FDA is approving new products in record-breaking time. As a result, managed care professionals are overwhelmed by new product introductions and are unable to perform consistent, comprehensive reviews. A resource providing consolidated clinical and strategic economic information that allows managed care professionals to manage these new products is needed.
Health care providers must confront in particular the economic aspect of advances in biotechnology. Establishing an appropriate balance between the business and clinical aspects of expensive yet valuable biotechnology compounds is difficult. Managed care providers are expected to assess not only the efficacy and safety, but increasingly, the economic value of new treatments. With more than 300 new biotechnology products expected to be approved within the next three to five years, how will their value be measured and their use justified? Clinical choices, based on the relative value of one drug over another, will need to include consideration of outcomes. Realizing a greater value at a lesser cost, regardless of the products or services, must be the ultimate goal.

For managed care providers, therefore, a long-term business plan is essential. It should have four phases. environmental scanning, strategy formulation, strategy implementation, and strategy analysis. ${ }^{15}$

\section{Environmental Scanning}

The first phase of a strategic planning process involves identifying the clinical and economic forces present in the internal and external health care environments. This model incorporates published data, clinical judgment, and forces both within an institution and in the external health care environment that affect demand for and access to new drug therapies. The following questions help to assess the internal environment.

- Does the institution market itself as offering state-of-the-art therapies?

- Do new biotechnology products fit within the institutional mission and provide a competitive advantage in the marketplace?

- Does the clinical staff adopt new therapies quickly and enthusiastically?

- Is the institution financially stable enough to invest in the latest treatment advances?

- Is the payor mix of the institution receptive to new drug therapies? 
The foremost consideration in the external environment is the need to monitor drugs in research and development-in particular, drugs likely to receive FDA approval that will be used often within the institution. Other factors that should be considered are therapeutic trends, reimbursement (including reimbursement for off-label uses), pharmaceutical benefit management programs with restrictive formularies, and pharmacoeconomic data.

Creating an efficient, central reference source that distributes information about new products and emerging pharmaceuticals in the retail, biotechnology, and oncology therapeutics areas is essential. This resource could serve as an industry standard, with all heaith care providers relying on it for analyses and reviews. The authors have recently proposed an Internet-based system that reports both external and internal trends similar to that developed by the Biotech Medical Management Association. ${ }^{16}$ To be effective, this system would have to provide access to current technical reviews, clinical research study results, and financial trending information. The system should also allow for custom use by managed care organizations, practicing physicians, employers, pharmaceutical manufacturers, and consumers.

\section{Strategy Formulation}

Clearly, managed care pharmacists are becoming increasingly responsible for prompt and accurate forecasting, and their ability to make decisions regarding pharmaceutical coverage is required. Managed care plans are being held accountable to multiple external forcesincluding government organizationsresulting in an ever-growing need for more sophisticated and comprehensive decision-making tools. Managed care pharmacists and medical professionals agree that the following actions are required: (1) be proactive and progressive, to monitor and manage more effectively the growing body of pharmaceuticals being approved rapidly ("fasttracked") by the FDA; (2) establish a standardized national tool to support formulary and medical/drug policy decision making; (3) help in planning resource allocation to avoid stalled and inconsistent decision making; and (4) recognize and monitor secondary costs associated with product use, and support off-label indications when appropriate.

One of the best methods of predicting the impact of a new therapy is by quantitatively forecasting demand, cost, and revenue. The demand for a new product can be predicted by estimating the number of patients with a particular medical indication and anticipated physician use of the suggested therapy. Total acquisition costs per year may be calculated by multiplying the quantitative demand (number of patients multiplied by doses per patient) by the acquisition cost per dose. Total acquisition costs also include preparation and administration costs. If the forecast projects high costs without revenue, one must consider using drug restriction policies, practice guidelines, and care pathways.

\section{Strategy Implementation}

Successful implementation of a strategic plan is the result of communication, education, and monitoring. The plan must be communicated to key physicians, clinical pharmacists, and nurses. Additionally, educational conferences and written summaries of the strategic plan can be used to inform staff and generate support for the plan. Finally, a monitoring process to provide feedback to clinicians and administrative personnel regarding the clinical and economic impact of a new therapy is vital.

Rapid and focused quality analysis of new products supported by documentation could be assured by using concise executive overviews and briefings. A central reference source could provide access to all analyses, reports, and current information and allow frequent updates. The lack of "barriers to entry" of an Intranet or Internet system would open real-time information access to office-based and large-group health care practices, remote clinics and field personnel, billing and customer relations, and even employers and patients. In addition, side-by-side product comparisons would offer clear clinical, economic, and humanistic points of comparison that are rarely available currently.

\section{Strategy Analysis}

The final phase in the strategic planning process is a dynamic component that evaluates forecasting accuracy, considers the effectiveness of clinical management approaches, and reveals drug performance in patients outside of a clinical trial. As new drug data become available, the plan may be modified accordingly. Reassessment of the strategic plan every three to six months is advisable, although no standard interval exists.

This early review of the analysis would require a set of benchmarks for both data recognition and evaluation The ideal design would be based on the previously proposed central reference source for formulary and medical policy decision making. A centralized strategy and rapid assimilation of new information would require that each product undergo the same rigorous evaluation.

A lack of peer-reviewed literature supporting the use of a new biotechnology drug or therapy at the time of product release creates a major dilemma. The requirement to use recently released clinical trial and published data leads to the need for a "quality of evidence" rating system to provide consistency and clarity among all products in the proposed information database. Data would then be rated according to predetermined benchmarks. The result would be access to data very early in the review process from sources otherwise unavailable through our current peer-review system. This central resource would serve an especially critical role in the emerging fields of pharmacoeconomic evaluations, cost utility, and quality-of-life analyses.

\section{Future Trends in Biotechnology}

Over the next two decades, discoveries in biotechnology and advances in gene ther- 
apy will transform the practice of medicine-the traditional treatment concepts of palliation, cure, and prevention will move toward human enhancement and capability. The drug discovery process will be even more accelerated, and new drugs entering the marketplace will be developed based on the biologic cause or pathway of human disease. Development of gene chips containing the DNA representative of all the human genes will facilitate individual gene profiling as well as analysis of distinctive disease-specific gene patterns. These chips will allow clinicians to make much more accurate diagnoses and recommend particular therapies with much greater certainty. In general, health care will evolve to a more sophisticated level of customization, enabling therapeutic selection precisely tailored to an individual's biochemistry. The advances expected to occur through biotechnology in the coming decade will have far-reaching effects on patients, clinicians, and payors, and will redefine the concept of medical practice in the new millennium.

\section{Conclusion}

The managed care pharmacist is charged with being responsible not only to the employers or health care plans, but also to the general public. Redeveloping partnerships between managed health care organizations and practicing clinicians or health care facilities will undoubtedly create opportunities for all parties. Moreover, creating strategic clinical networks between key pharmaceutical and biotechnology product manufacturers will allow greater opportunities for onsite research and critical input regarding the products most needed in clinical care.

\section{References}

1. Zajtchuk $\mathrm{R}$. New technologies in medicine: biotechnology and nanotechnology. Disease-amonth. St. Louis, MO: Mosby, Inc, 1999: 452-92.

2. Itakura $\mathrm{K}$, et al. Expression in Escherichia coli of a chemically symthesized gene for the hormone somatostatin. Science 1997; 198: 1056-63.

3. Anonymous. Human insulin receives FDA approval. FDA Drug Bull 1982; 12: 18-19.

4. Emst \&r Young. LLP. 13th Biotechnology Industry Annual Report, 1999.

5. Wiley SR. Genomics in the real world. Current Pharmaccutical Design 1998; 4: 41.7-22.
6. Saunders NJ, Moxon ER. Implications of sequencing baclerial genomes for pathogenesis and vaccine development. Curr Opin Biotechnol 1998; 9: $618-23$

7. White R. Technology and the evolution of human genetics. Exp Cell Res 1.999; 253: 305-07.

8. Gotschalk U, Chan S. Somatic gene therapy. Present siluation and future perspective. Drug Res 1998; 48: $1111-20$

9. Mahato RI, Smith LC, Rolland A. Pharmaceutical perspectives of nonviral gene therapy. Adv Genet 1999; 41: 95-156.

1.0. Levitzki A. Targeting signal transduction for disease therapy. Curr Opin Cell Biol 1996;8. 239-44.

11. Bode $W$, el al. Structural propertics of matrix metalloproteinases. Cell Mol I_ife Sci 1999; 55 $639-52$

12. Kugler $A$. Matrix metalloproteinases and their inhibitors. Anticancer Res 1999; 19: 1.589-92.

13. Galderisi U, Cascino A, Giordano A. Antisense oligonuclcotides as therapcutic agents. J Cell Physiol 1999; 181: 251-57.

14. Klohs WD, Hamby JM. Antiangiogenic agents. Curr Opin Biotechnol 1999; 10: 544-49.

15. Huber SH, Kirschenbaum B. A stepwise approach to strategic planning for biolechnologyproduced drug products: a guide for pharmacisis Scientific Therapeutics, Inc. 1991

16. Biotech Medical Management Association. Bio Assessment Technologies. Available at: hup://www bioaslec.com. Accessed June 2, 2000. 